\title{
JOURNAL OF OBJECT TECHNOLOGY
}

Online at www.jot.fm. Published by ETH Zurich, Chair of Software Engineering @JOT, 2003

Vol. 2, No. 3, May-June 2003

\section{Proving Pointer Program Properties Part 2: The Overall Object Structure}

Bertrand Meyer, ETH Zurich, Switzerland and Eiffel Software, Santa Barbara, USA

\begin{abstract}
The run-time object structure of object-oriented programs typically relies on extensive use of references (or pointers). This second part of a general mathematical framework for reasoning about references handles the overall properties of the structure, not distinguishing between individual links but only considering whether any reference exists between two objects. It provides a basis for dealing with memory management and especially garbage collection.
\end{abstract}

This is part of a series of articles. See here for part 1.

\section{BASICS OF THE RELATION MODEL}

The coarse-grained model of object structures developed here will rely on a relation between objects. For this reason we call it the relation model; the finer-grained models of the subsequent articles, which take into account individual attributes of classes, and hence individual fields of objects, will expand this relation into a set of functions.

\section{Addresses and objects}

The execution of an object-oriented system creates and manipulates objects. Each object is stored at a certain address, and we can only use a finite set of addresses. We express this by introducing a constant, the set of addresses:

\section{[A1] Addresses: $\mathbb{F}(\mathbb{\mathbb { N }})$}

where $\mathbb{N}$ is the set of natural integers and $\mathbb{F}(X)$, for any set $X$, is the set of all finite subsets of $X$ (a subset of $\mathbb{P}(\boldsymbol{X})$, the powerset of $X$, which contains all its subsets). Property [A1] specifies Addresses as a finite set of integers.

This property is an axiom asserting the existence of a distinguished member, Addresses, of a known set IF ( $\mathbb{N}$ ); the colon ":" recalls the type declarations of some programming languages. 
An element of Addresses represents a potential object; only at certain addresses will we find actual objects. We represent this observation by introducing an explicit variable subset of Addresses:

\section{[A2] Objects: $\mathbb{F}(\mathbb{N})$}

with the property that

[I3] Objects $\subseteq$ Addresses

[I3] is an invariant; we will have to prove that every event preserves it.

A point of modeling style, also applicable to later axioms and invariants: it would be possible to avoid the invariant [I3] altogether by defining the set Objects directly, in the axiom [A2], as a member of $\mathbb{I F}$ (Addresses) rather than $\mathbb{E}(\mathbb{N})$. The practical effect is the same: we would still have to prove that any element added to Objects by any event is in Addresses; this is a type check rather than an invariant preservation proof. Making the invariant explicit is clearer.

The choice of natural integers - IF ( $\mathbb{N})$ - for Addresses, and as a consequence for objects, deserves a justification. It is legitimate to restrict ourselves to a finite set of addresses since this is what we will have on any actual computer or bank of computers; representing them by integers does not imply any implementation commitment but simply reflects the concept known as object identity: each object created during execution has a separate identity, even if its content happens to be identical to that of another object. Each element of Addresses represents such a unique identity for a possible object; it does not have to represent a physical address in the memory of a computer. We will explore later the more precise properties of Addresses and its possible relation to actual memory addresses ${ }^{1)}$.

It will be useful to give a name to addresses not occupied by objects:

[D4] Unused $\triangleq$ Addresses - Objects

where - is set difference. ( $\triangleq$ means "is defined as.")

\section{Linking objects}

The topic of our study is the set of reference links that may exist between objects. At the highest level of abstraction, we represent it by a relation

${ }^{1}$ See "REPRESENTING ADDRESSES", 4, page 99 
[A5] attached: $\mathbb{N} \leftrightarrow \mathbb{N}$

where $A \leftrightarrow B$ is the set of relations between any two sets $A$ and $B$. (More precisely we are interested in $\mathbb{N} \leftrightarrow \mathbb{N} \mathbb{N}$, the set of finite relations, but this makes no difference since Addresses itself is finite, so all relations on it are finite.)

Names of sets of addresses and objects, as Addresses and Objects, start with an upper-case letter; names of functions, relations and predicates, as attached, start with a lower-case letter.

The informal meaning of attached is that it contains a pair $[01,02]$ to reflect that there is a reference from the object at 01 to the object at 02 . The name attached reflects the Eiffel terminology, which says that at run time a reference may be "attached to" a certain object.

This model - called from now on the Relation Model - provides a good basis for our study. At run time your program has created a set of objects. Each object, stored at a certain address, is made of a number of fields; a field may be of an "expanded" type, meaning that it is a directly usable value - an integer, a character ... - or it may be a reference. A reference either takes us to another object or is "Void":

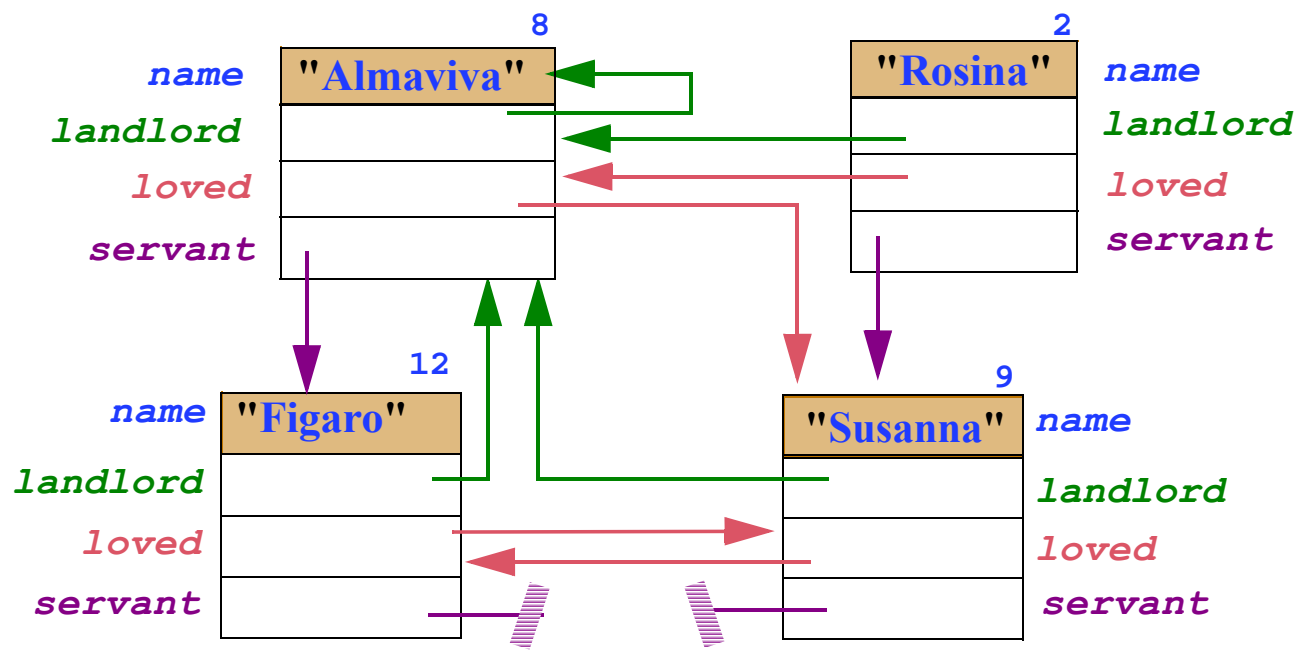

Since this discussion focuses on the references, we ignore the actual object contents (the expanded fields). The relation attached defines a graph of references between objects. Ignoring individual fields, it only indicates whether at least one reference exists between any two objects; it is represented, for the object graph of the preceding figure, by the bold lines on the next figure. If two fields of an object are references to the same object (as landlord and loved from the object labeled 2 to the object labeled 8), they yield only one link in the relation. 


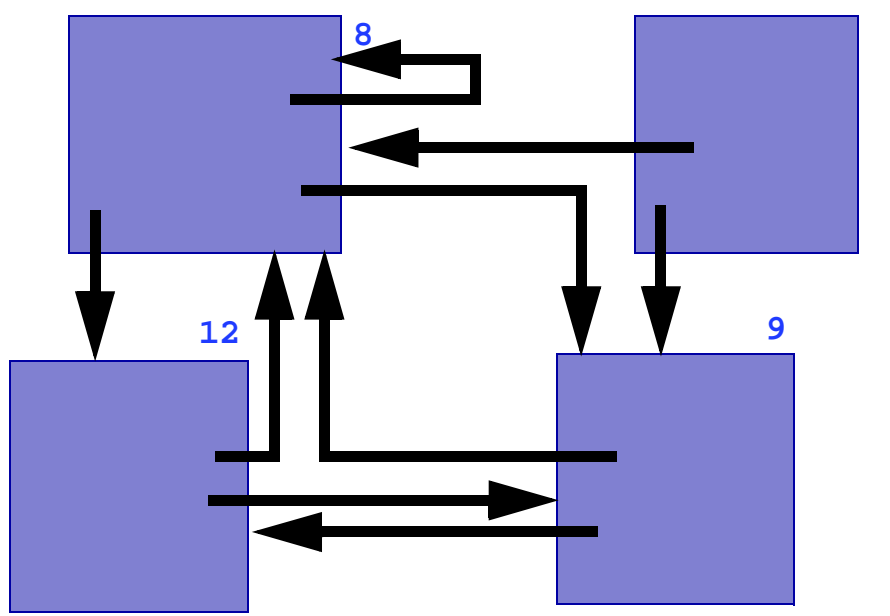

By considering only the relation attached, we disregard individual attributes such as Iandlord and Ioved; to get a realistic model we will have to reintroduce them ${ }^{2)}$. Until then we can use attached as a coarse-grain view of the object structure, already sufficient to obtain a remarkable set of properties.

The model defines attached as a relation, meaning a set of pairs; that a particular pair of addresses [ $i, j]$ belongs to the relation (in symbols, $[i, j] \in$ attached) means that there is a reference from the object at $i$ to the object at $j$. With the addresses shown, the relation illustrated is the set of pairs $\{[8,8],[8,9],[8,12],[2,8],[2,9]$, $[12,8],[12,9],[9,8],[9,12]\}$.

\section{Void links}

Every practical programming language that offers references has a notion of "void" or "null" reference, used in particular to terminate chains of references in linked structures. In Eiffel, Void also serves as default initialization value for reference types (like false for BOOLEAN and zero for INTEGER).

The Self language tried to do away with Void, but the result seems to confirm the need for this concept.

How do we model void? We don't. One of the benefits of using relations and partial functions is to spare us the need for any special element to represent Void. If a reference from obj is void, the corresponding function, a subset of attached, will simply not be defined for obj will not contain any pair of the form $[o b j, x]$ for any $\mathbf{x}$. The preceding figure illustrates this for objects 9 and 12 ("Figaro" and "Susanna") and function servant. This convention is all we need; it will be invaluable when we prove properties of data structures in later articles.

\footnotetext{
${ }^{2}$ See part 3 of this article series.
} 


\section{The Basic Object Constraint}

We require the relation attached to satisfy a fundamental invariant, the Basic Object Constraint:

[I6] attached $\subseteq$ objects $\leftrightarrow$ objects

which expresses that links only exist between objects, not arbitrary addresses. The Basic Object Constraint is the combination of two separate properties:

$$
\begin{aligned}
& \text { [T7] domain }(\text { attached }) \subseteq \text { objects } \\
& \text { [T8] range }(\text { attached } \subseteq \subseteq \text { objects }
\end{aligned}
$$

where, if $r$ is a relation, domain $(r)$ is its domain, the set of elements $\mathbf{x}$ such that $r$ contains a pair of the form $[x, y]$ for some $y$; and range $(r)$ is its range, the set of $y$ such that $r$ contains a pair of the form $[\mathbf{x}, \boldsymbol{y}]$ for some $\mathbf{x}$.

The model could define [T7] and [T8] as independent invariants and deduce the Basic Object Constraint

[I6] as a theorem; we choose to do the reverse, taking the more complete property as the invariant.

The first part [T7] of the invariant, domain (attached) $\subseteq$ objects, states that all references to objects come from other objects: there's no one out there keeping references to our objects. We accordingly call it the No Big Brother property. It will be essential for modeling dynamic object allocation ${ }^{3}$, a cornerstone of programming with dynamic data structures. Any event that creates an object must be able to use any unallocated memory address, meaning any element of the set Unused (defined above as Addresses - objects). Without the No Big Brother property such an element could contain links to objects; making it part of objects might then add spurious reference links to the object structure, destroying its consistency and causing trouble for memory management, especially garbage collection.

Maintaining this property invariant will impose a constraint on memory deallocation ${ }^{4)}$ as performed by a garbage collector (GC): when reclaiming a member of objects to return it to Unused, the GC will need to erase (or "zero out") all its outgoing links, to satisfy this clause and enable a later memory allocation event to reuse it without risk.

The second part [T8] of the Basic Object Constraint, range (attached) $\subseteq$ objects, states that if, from an object, we follow a reference, we get an object. We'll call it the No Zombie property, using the definition

[D9] Zombies $\triangleq$ range (attached) - objects

3 "Object creation", page 88.

4 "Full garbage collection", page 91. 
The Basic Object Constraint prohibits zombies through the following restatement of [T8]:

$$
\text { [T10] Zombies }=\varnothing
$$

The Basic Object Constraint must be our obsession when we write software in languages meant for manual memory management such as $\mathrm{C}$, Pascal, Ada and $\mathrm{C}++$, where the definition of disaster is to end up with a Zombie object that is referenced by other objects but not known any more as a member of the community of objects. This happens because at some point our program has "freed" the memory allocated to the object (or "disposed" of it in Pascal terminology) even though some non-zombie, somewhere, somehow, still keeps a reference to it.

A number of companies exist primarily to provide tools that help developers debug programs (often in $\mathrm{C}$ and $\mathrm{C}++$ ) that do not satisfy the Basic Object Constraint, so that promoting that constraint might in the current market conditions appear anti-business. Any advances reported here are, fortunately, of a preliminary nature only.

In a language supported by automatic memory management, such as Eiffel, the Basic Object Constraint is also an obsession, but sensibly transferred from the application programmers to the authors of the memory management system, especially the garbage collector (GC).

The No Zombies property has two other consequences presented later: a particular obligation on incremental garbage collection ${ }^{5)}$; and, if we understand Addresses to represent actual memory addresses, a rejection of references to subobjects, discussed when we look at how to provide a concrete interpretation for the set Addresses.

The Basic Object Constraint defines the fundamental invariant under which the GC will pursue its goal of returning to Useless any obsolete elements of objects: in this process, it must create neither Big Brothers nor Zombies.

Rather than [T8] or [T10], we will mostly use the No Zombie property under yet another form:

$$
\begin{aligned}
& \text { [T11] attached }(\cdot \text { objects } \cdot) \subseteq \text { objects } \\
& \text {-- Basic Object Constraint: }
\end{aligned}
$$

where, for any relation $r$ and a subset $X$ of its source set, $r(\cdot X \cdot)$ is the image of $X$ under $r$ : the set of elements $y$ such that $r$ contains a pair of the form $[x, y]$ for some member $\boldsymbol{x}$ of $x$. Although equivalent to [T8], this form takes advantage of the image operator, which enjoys such pleasant properties as

5 See "Incremental garbage collection", page 96, and "REPRESENTING ADDRESSES", 4, page 99 . 
-- For any subsets $\boldsymbol{X}$ and $\boldsymbol{Y}$ of the source set of $\boldsymbol{r}$ and $\boldsymbol{s}$ :

[T12] $\quad x \subseteq Y \Rightarrow r(\bullet X \bullet) \subseteq r(\bullet Y \bullet))$

[T13] $r \subseteq s \Rightarrow r(\bullet X \bullet) \subseteq s(\bullet X \bullet))$

[T14] $r^{-1}(\cdot \varnothing \cdot)=\overline{\operatorname{domain}(r)}$

[T15] $(r \cup s)(\bullet X \bullet)=r(\bullet X \bullet) \cup s(\bullet X \bullet)$

[T16] $r(\cdot X \cdot) \subseteq$ range $(r)$

[T17] $r^{+}(\cdot X \cdot) \subseteq$ range $(r)$

[T18] id $[X] \subseteq r \Rightarrow r^{*}(\cdot X \cdot) \subseteq$ range $(r)$

and more to come. These use the following notations: $\varnothing$ is the empty set; $x$ is the complement of a set $X ; i d[X]$ is the identity relation on $X$; if $r$ is a relation, $r^{-1}$ is the inverse relation of $r, r^{+}$its transitive closure, and $r *$ its reflexive transitive closure.

The discussion will rely extensively on the image operator, which lets us treat a relation as a function from subsets to subsets, and hence take advantage of all the notations and properties of functions, more convenient than those of general relations.

\section{STACK, HEAP, GARBAGE AND LIVE OBJECTS}

In an object store, some objects are "live" and other are "garbage". The live objects are those reachable, directly or indirectly, from "root" objects.

\section{Stack and heap}

Following the common structure of O-O programming language implementation, we refer to the set of root objects as "The Stack" and introduce it explicitly as a variable:

\section{[A19] Stack: $\mathbb{F}(\mathbb{N})$}

We require the stack to satisfy two properties:

- Since the model treats all data as objects, the stack may only contain objects. Some of the values on the stack may be very simple, for example a single integer (of no interest at this stage since we ignore non-reference data) or a single reference, but we model them as objects all the same.

- We exclude any references leading to stack objects, although references may exist from stack objects to others, called "heap" objects: 


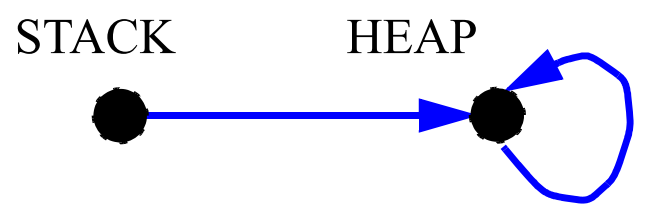

The second property does not hold in $\mathrm{C}++$, with its arbitrary $\mathrm{C}$-style pointers, but Eiffel and some other object models observe it because it makes programming simpler and safer.

The combination of these properties is our third invariant:

[120] Stack $\subseteq$ objects - range (attached)

Together with [13], this invariant implies:

[T21] range (attached) $\cap$ stack $\subseteq \varnothing$
[T22] stack $\subseteq$ objects
[T23] stack $\subseteq$ Addresses

We may now define the heap:

[D24] Heap $\triangleq$ Objects - Stack

implying that

$$
\begin{aligned}
& \text { [T25] Heap } \subseteq \text { objects } \\
& \text { [T26] Heap } \cap \text { Stack }=\varnothing \\
& \text { [T27] objects }=\text { Stack } \oplus \text { Heap } \\
& \text { [T28] range (attached) } \subseteq \text { Heap }
\end{aligned}
$$

Here $\oplus$ denotes disjoint union of subsets; a property of the form $A \oplus B=C$, such as [T27], stands for two separate properties: $A \cap B=\varnothing$ and $A \cup B=C$. [T27] follows from [D24] and [T22]. Together with the No Zombies theorem [T8], [T27] implies range (attached) $\subseteq$ stack $\oplus$ Heap, from which [T21] yields the theorem [T28]. This theorem explains the importance of the heap. 
Using the image operator we may draw a set of consequences from the preceding properties:

$$
\begin{aligned}
& \text { [T29] attached }(\cdot \text { objects } \bullet \subseteq \text { Heap } \\
& \text { [T30] attached }(\bullet \text { Heap } \bullet \subseteq \text { Heap } \\
& \text { [T31] attached }(\cdot \text { objects } \bullet \subseteq \text { Heap } \\
& \text { [T32] attached* }(\cdot \text { Heap } \bullet \subseteq \text { Heap }
\end{aligned}
$$

[T29] uses [T16] to restate [T28]. [T30] follows from [T29] through the subsetting property [T12] of images. The last two theorems follow from the previous two by iterating the same reasoning.

This proof of [T31] and [T32] is a fixpoint proof (generalized proof by induction) in the following sense: if for a certain predicate $p$, a certain relation $r$ and a certain set $A$ we can show that $p$ (A) holds, and moreover that whenever $p(X)$ holds $p(r(\bullet X \bullet))$ also holds, then we may deduce that $p \quad\left(r^{*}(\bullet A \bullet)\right)$ holds. This is also how [T17] and [T18] follows from [T16].

\section{Live and garbage objects}

From roots (the stack) we define live objects, those reachable from the stack directly or indirectly:

$$
\text { [D33] Live } \triangleq \text { attached* ( Stack })
$$

as illustrated by the following informal figure of the object store (next page), whose details will soon be completely clear.

Some properties follow immediately from the definition of Live [D33]:

$$
\begin{aligned}
& \text { [T34] Stack } \subseteq \text { Live } \\
& \text { [T35] Live } \subseteq \text { Objects } \\
& \text { [T36] attached }(\bullet \text { Live }) \subseteq \text { Live } \\
& \text { [T37] attached* }(\bullet \text { Live }) \subseteq \text { Live }
\end{aligned}
$$

[T34] comes from the general property of closures that id [X] $\subseteq r^{*}$ for any relation $r$ of source set $\boldsymbol{X}$. [T35] results from the combination of [T22], the closure property of images [T18], and the No Zombie property [T8]. [T36] follows from taking the image by attached of both sides of [D33] and using the general property that $r$ composed with $r$ is a subset of $r *$ [T37] takes [T36] to its fixpoint using [T18]. 


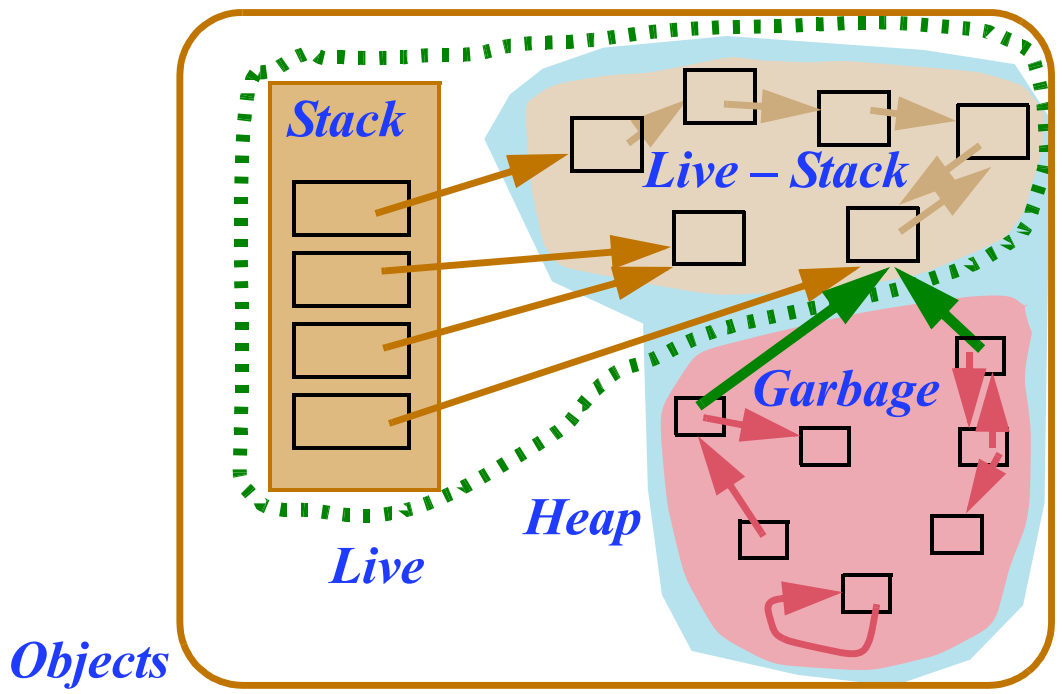

Outside of Live we find the non-reachable objects, known more prosaically as garbage (hence "Garbage Collector"):

[D38] Garbage $\triangleq$ objects - Live

sometimes more convenient to use under the form

$$
\text { [T39] objects }=\text { Live } \oplus \text { Garbage }
$$

which follows from [T35], and itself implies

$$
\begin{aligned}
& {[\text { T40] }} \\
& \text { Garbage } \cap \text { Stack }=\varnothing \\
& {[\text { T41] }} \\
& \text { Garbage } \subseteq \text { Heap }=(\text { Live }- \text { Stack }) \oplus \text { Garbage } \\
& {[\text { T43] } \text { objects }=\text { Stack } \oplus(\text { Live }- \text { Stack }) \oplus \text { Garbage }} \\
& {[\text { T44] attached }-1(\cdot \text { Garbage } \cdot) \subseteq \text { Garbage }}
\end{aligned}
$$

Again you may follow all these properties on the preceding figure. [T40] is a consequence of [T34] and [D38]. [T41] restates [T40] based on [D24]. [T42] restates [D24] using [T39] and [T40]. Then [T43] combines [T27] and [T42]. [T44] states that links into Garbage may only 
come from Garbage; this is a consequence of [T36] and [T39]. We may not, however, infer the symmetric property attached ${ }^{-1}(\bullet$ Live $\cdot) \subseteq$ Live: as illustrated by the bold dark-green links on the last figure, there may be links from Garbage to Live.

The purpose of a GC is to remove all or some of Garbage. The inverse of [T35], objects $\subseteq$ Live, means that there is no garbage. It's not an invariant, but it will be the goal (the postcondition) of a full GC cycle. Let's give this property a name:

[D45] No_garbage $\triangleq$ Live = objects

[T29], [T30] and [T36], and their fixpoint variants indicate that each of the sets objects, Heap and Live is "stable" under attached and hence its closure, calling $A$ stable under $r$ if $r(\cdot A \bullet) \subseteq A$. The set $S t a c k$, on the other hand, is clearly not stable under attached; neither is Garbage because of the possible presence of links from Garbage to Live (the just noted dark-green links).

\section{Invariants so far}

As we will now consider events that may affect the state, and must prove that each of them preserves every invariant, it is useful to collect invariants seen so far:

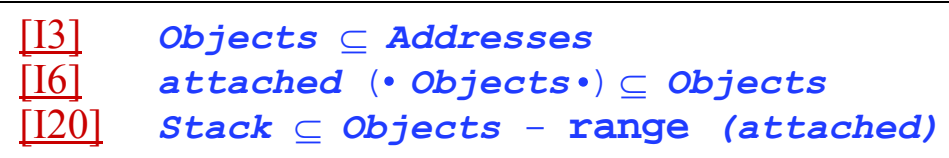

\section{OBJECT CREATION AND DESTRUCTION}

Equipped with a model of the object structures, we now move on to a description of the the basic memory management operations that may affect them: object creation, stack allocation, and object deletion including full and incremental garbage collection.

All will be specified as B-like events, i.e. substitutions that occur under the control of a certain guard, with an Eiffel-like syntax.

I use this syntax because I'm more comfortable with it, but don't be misled by appearances, this is not programming, it's mathematical specification in B style. 


\section{Object creation}

Our first event models a basic instruction of an O-O language, of the following form

\section{create new}

which creates a new object and attaches it to new.

Here new is just a variable name, not a keyword. The corresponding to the $\mathrm{C}++/ \mathrm{Java} / \mathrm{C} \#$ syntax would be new_ = new TYPE_OF_NEW ();

Here is the corresponding mathematical event is:

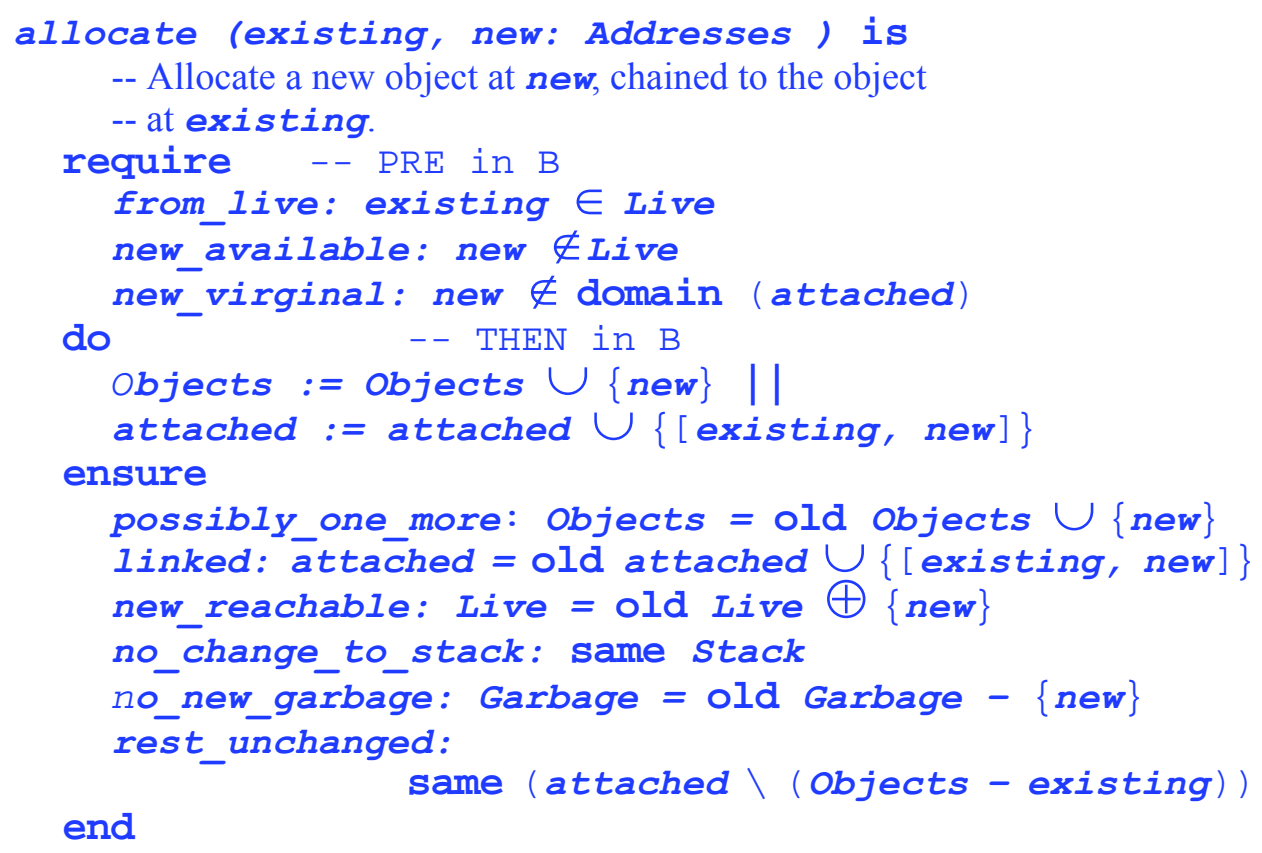

The do clause contains two state changes, one affecting objects and the other attached; they are separated by the symbol || to indicate parallel execution. (Another B notation, $x, y$ $:=e, f$ for multiple simultaneous assignments, would also work here.)

The postcondition uses the following two notations:

- same $x$ is an abbreviation for $x=$ old $x$, expressing that the event doesn't change the value of $\boldsymbol{x}$.

- " $\backslash "$ denotes restriction: $r \backslash A$, for a relation $r$ and a subset $A$ of its source set, is the relation made of all pairs $[i, j]$ in $r$ whose first element $i$ is in $A$.

So the clause rest_unchanged expresses that the event doesn't change anything in the relation attached except for the new link at existing. 


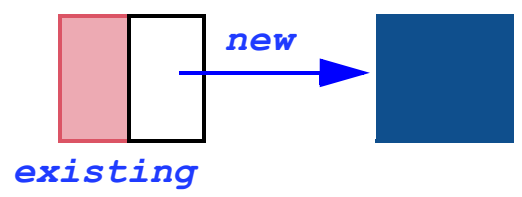

The event's argument existing and the precondition clause from_live reflect the property that in object-oriented programming, as illustrated abovee, it is only possible to create an object from another object, which must be live: the instruction create new will be part of a class text, and will be executed on behalf of some instance of that class; if the object were not reachable, execution would have no way to get to that instruction. If new is an attribute ${ }^{6)}$ of a class, existing is in the Heap; if new is a local variable of a routine, existing is an element of the stack.

The event uses new as an argument, denoting the abstract address of the new object. In OO languages you don't specify new, so we could remove this argument and instead use an integer outside of Live, chosen non-deterministically to satisfy the precondition. It is simpler to make new explicit.

The last two precondition clauses state that we may only reuse an address if is satisfies the following requirements:

- It doesn't host a live object (new_available).

- It's not in the domain of attached, that is to say, it doesn't contain a link to any object (new_virginal).

The next section discusses these two clauses further ${ }^{7)}$. Let us now prove that the event allocate ensures its postcondition and preserves the invariants:

- The first two clauses of the postcondition, possibly_one_more and linked, simply restate the event's definition.

- For new_reachable: we are only adding one link, the pair [existing, new], to the relation attached, so any object in Live will remain in it. Adding the link implies adding new to attached ( $\bullet$ ive•) and hence, from [T36], to Live itself. The second precondition clause, new_available, guarantees that this is a disjoint union. The only other way to add objects to $L$ ive would be through links from new to some other addresses (which would have had to be garbage or non-objects before the event); this is impossible because of the precondition clause new_virginal, without which we couldn't do this part of the proof.

- The clause same_stack is trivial since the event doesn't modify stack.

- The clauses same_garbage and rest_unchanged follow from the preceding clauses.

${ }^{6}$ Also called data member (C++), instance variable (Smalltalk), field (.NET).

7 "Getting the precondition right", page 90. 
- The invariant [I3] stated $^{8)}$ objects $\subseteq$ Addresses; its preservation follows from the postcondition clause possibly_one_more since new $\in$ Addresses.

- The invariant [I6] stated ${ }^{9)}$ attached $\subseteq$ objects $\leftrightarrow$ objects. The postcondition clause linked tells us that the event may add at most one element, existing, to its domain, existing, and at most one element, new to its range; this preserves the invariant since both will be members of objects.

- The invariant [I20] stated $^{10)}$ stack $\subseteq$ objects - range (attached). Since the event does not change stack and does not remove any element from objects, it could only invalidate this property by adding a member of stack to range (attached). This means adding to attached a link leading into the stack. But the only new link leads to new, which from clause new_available is not in Live, and hence from [T34] not in Stack.

\section{Getting the precondition right}

The history of the two precondition clauses new available and new virginal is instructive. Initially I used a single clause new $\notin$ objects, meaning that the allocation uses a memory cell at a fresh address. The correctness proof was straightforward. This form, however, is stronger than required: if we find that an object is in Garbage, it's OK to reuse its cell.

So I replaced the clause new $\notin$ Objects by new $\notin L i v e$, called new_available above. But then for the new reachable postcondition clause, Live $=$ old $L i \overline{v e} \oplus\{n e w\}$, I could not prove that the right-hand side is a subset of the left-hand side. The reason, it turns out, is that it is not always legitimate to recycle a garbage object new. If new has a link to another garbage object go, recycling new will make go — and possibly other objects as a result - reachable again, so the new Live will contain more than just old Live $\oplus$ \{new\}. Indeed we may only reuse a garbage object if it contains no links to other objects. The precondition clause new virginal takes care of this, permitting the above proof.

This illustrates that even though the discussion relies on straightforward concepts t's still possible to make serious mistakes, which an attempt at mathematical proof will uncover.

The consequences are not just theoretical. In a GC, failing to observe new_virginal is a real bug: failing to zero out a memory cell before recycling it. If the cell contains a link to a garbage object go, this will suddenly bring go back like Lazarus to the realm of the living. But a link to a live object 10 - such as the dark green links in the earlier figure ${ }^{11}$ - is just as bad since 10 will now have a spurious incoming link, preventing it from being reclaimed if, later on, all legitimate links to it disappear. Such a bug causes inexorably growing "memory leaks" and has plagued more than one released GC.

\footnotetext{
${ }^{8}$ Page $\underline{78}$.

${ }^{9}$ Page $\underline{81}$.

10 Page 84 .

11 Page 94 .
} 
The theoretical difficulty goes away if we replace new available and new virginal by the stronger single clause new $\notin$ objects. This would match the practice of $\mathrm{O}-\mathrm{O}$ language implementations: it's fairly easy to keep track of the set of all objects, whereas finding allocated objects that are not in $L i v e$, meaning they are in Garbage, usually requires performing a garbage collection; but then, having found the garbage objects, you might just as well remove them not just from Garbage but from objects too. The event col lect_al I, introduced next, does this. Here we retain the weaker form of the precondition, since it is permissible, as we have just proved, to reuse any garbage object that you have been able to uncover, provided you zero it out to satisfy new virginal and preserve [T7] (part of the invariant [I6]). This weaker precondition will become directly relevant when we consider incremental garbage collection, as modeled by another event, collect_some ${ }^{12)}$.

\section{Full garbage collection}

Although we'll need to model the other form of allocation (on the stack) ${ }^{13}$ ), for the moment we remain in the heap to examine events that deallocate objects, including both full GC and the freeing of individual garbage objects.

The following event represents a full garbage collection cycle:

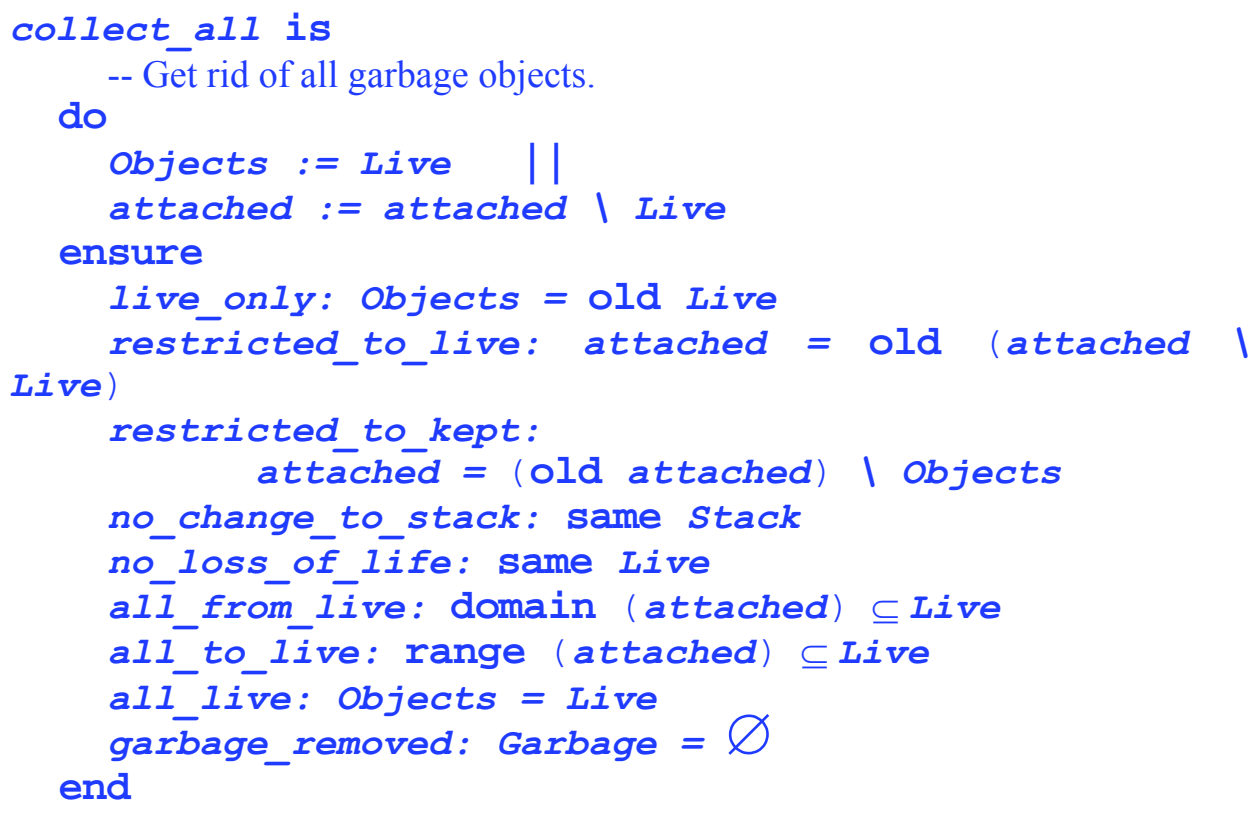

12 Page 96.

13 Event allocate_on_stack, page $\underline{98}$. 
In the second assignment the replacement for attached is attached I Live, denoting attached restricted to its pairs [i, $j]$ for which $i \in$ Live; or, equivalently (see [T39]), attached deprived of all pairs for which $i \in$ Garbage.

The purpose of garbage collection is to restrict the set of objects to live ones, so the first assignment may seem sufficient; but if we didn't also restrict attached we might produce Big Brother elements in Unused, which keep references to objects without themselves being in Objects. Then the allocate event wouldn't be able to recycle such elements of Unused as objects, since they wouldn't satsify its precondition clause new_virginal as just discussed. It is precisely to avoid polluting the address space with such wasted elements that we introduced the property domain (attached) $\subseteq$ Objects [T7] into the invariant [I6]; to preserve it, the event collect all must update the relation attached in addition to the set Objects.

Let's prove that the event ensures the postcondition clauses and preserves the invariants. The first two clauses of the postcondition, Iive_only and restricted_to_live, restate the event's definition. The next one restricted to kept is a direct consequence of these two. The clause no_change_to_stack is trivial since the event doesn't affect Stack.

The clause no loss of life states that the event collect all does not change the set Live, defined [Dי 33$]$ as attached* - ${ }^{*}$ stack $\left.\cdot\right)$, meaning stack $\cup$ attached $(\cdot \operatorname{stack} \bullet) \cup$ attached $(\cdot \operatorname{attached}(\cdot \operatorname{stack} \bullet) \cdot) \cup \ldots$ Since the successive sets in this union are all in Live and the event doesn't change attached on Live, it follows that Live itself is not changed.

The clause all_go_from_live is an immediate consequence of live_only. For all lead to live, assume a member $x$ of range (attached) that is not in $\bar{L}$ ive, and hence, because of no_loss_of_life, not in old Live. Relation attached must contain a pair of the form $[i, x]$ for some $i$ which, because of all_go_from_live, must be a member of Live, that is to say (again because of no_loss_of_life) old Live. Such a link from an object in old Live to an object not in old Live - a link from a live object to a garbage object, which must have existed before the event — is impossible as it would contradict [T36].

The property all_live follows from live_only and no_loss_of_life. The last clause garbage_removed is an immediate consequence.

If the invariant [16], attached $\subseteq$ objects $\leftrightarrow$ objects, holds before the event, it will still hold afterwards as an immediate consequence of the postcondition clause restricted to kept. Note that this is only because we require the event, through its second assignment, to zero out all attached links in the garbage objects that it reclaims - making them, as noted, available for later recycling by the event allocate.

Since the event doesn't change stack, it could invalidate the invariant [120], stack $\subseteq$ objects - range (attached), in one of only two ways:

- By removing from objects a member of stack; this is impossible because the postcondition clause only_live_kept tells us anything removed from objects must have been outside of Live, and so, by [T34], outside of Live.

- By adding to range (attached) an element of Stack; this is impossible too since Iive_only tells us the event doesn't add any link to attached. 
The reader, it is hoped, appreciates the eschatological significance of what has just been achieved. No later than page $\underline{93}$, we have managed to prove that if there is garbage it is all right to remove it.

\section{The free list}

Practical garbage collectors do not always return the objects they collect to Unused, that is to say, to the operating system. In fact, only the best GCs achieve this; this means for example that on many versions of Unix they can't rely on the standard C routine free, which instead of releasing a cell from the memory of the current process simply adds it to a special data structure, the free list, from where it is available for reuse by the same process. Then no matter how many free operations you have put in your program, its process space will not shrink. Only if the GC uses special primitives such as the Unix sbreak will the process actually relinquish memory.

This observation suggests that to maintain the realism of our model we should include a variant of the collect_all event that, instead of moving garbage away from the set of objects to Unused addresses, adds garbage objects to a special set Free. We'll have two new events: free_all, which adds garbage to Free without removing them from objects; and deallocate which returns the elements of Free to Unused. Combining these two events in sequence must have the same effect as collect_all.

Two main advantages follow from this extension to our model:

- We now have the flexibility of describing a GC that truly frees memory (collect_all), or can only return garbage to a free list (free_all), or returns garbage to the free list but occasionally deallocates the free list in sbreak style (free_all plus deallocate).

- We can now model incremental garbage collection. As will be seen in the next section, this would be impossible without the notion of a free list, because returning a subset of Garbage to Unused would violate the No Big Brother property. The introduction of Free permits a variant of free_all that collects some but not all of the garbage.

We introduce the free list as a variable:

\section{[A46] Free: $\mathbb{F}(\mathbb{N})$}

Elements of Free must be objects (otherwise we can't have links to them without violating No Big Brother); the only place where we can meaningfully have them is garbage, hence an invariant:

[147] Free $\subseteq$ Garbage 
In addition, elements of Free must be immediately reusable for the allocation of new objects — that's their whole raison d'être —, so they must satisfy the precondition clause new virginal of the allocate event ${ }^{14)}$ by not having any outgoing links, a condition we express through another invariant:

$$
\text { [I48] Free } \cap \text { domain }(\text { attached })=\varnothing
$$

In practice this means that any event that adds elements to Free must remove their outgoing links. The following figure illustrates the situation: it shows the set Garbage, extracted from the earlier picture of the overall object structure, with its new subset Free:

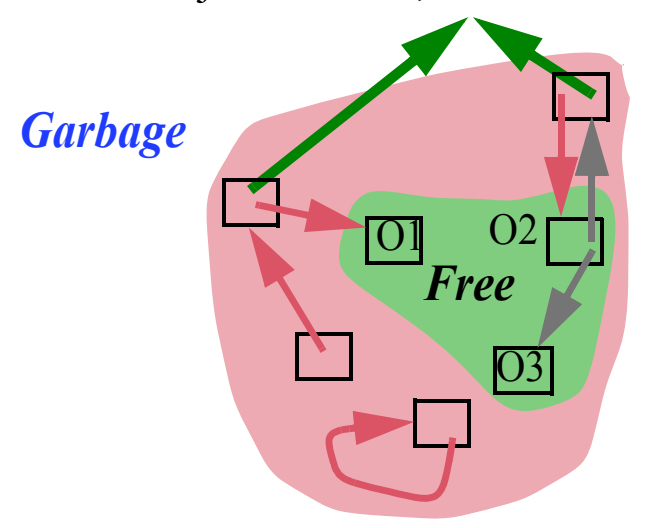

As per [I48], all outgoing links have been removed from the objects in Free; but there may still be incoming links into these objects, such as the red links into O1 and O2. Such links may only come from non-free garbage as per the following theorem:

$$
\text { [T49] } \text { attached }^{-1}(\cdot \text { Free }) \subseteq \text { Garbage - Free }
$$

Proof: incoming links into Free may not come from Free because of [148]; they may only come from Garbage because of [T44]. We may in fact infer from this a stronger property (although [T49] will be the useful form):

$$
\text { [T50] attached }{ }^{-1}(\cdot \text { Garbage } \bullet) \subseteq \text { Garbage - Free }
$$

Since the previous events did not involve Free, they all preserve our two new invariants.

The new event free_all — which will also preserve them — is almost identical to collect_all (the specification below highlights the differences) but returns freed elements to Free rather than Unused:

14 Page 88 . 


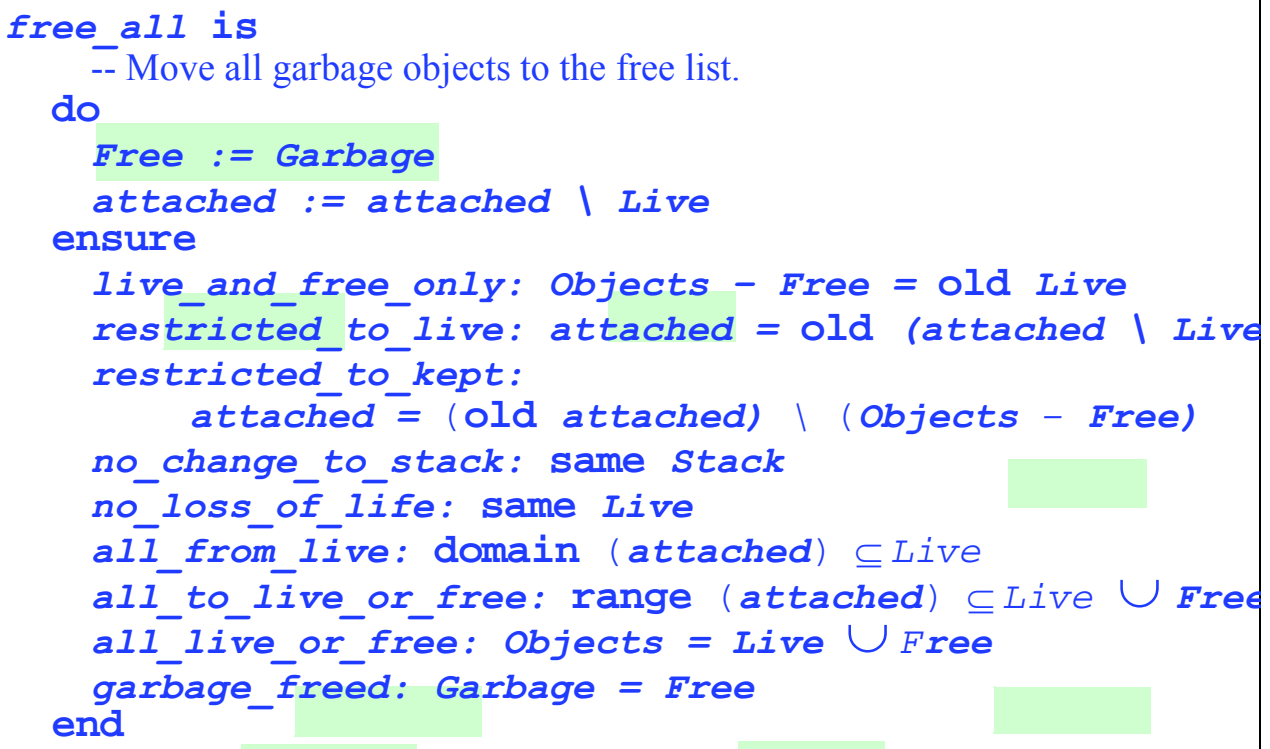

Proofs of the postcondition and previous invariants are the same as for collect_all; proofs for the two new invariants are immediate. As illustrated on the last figure, the state resulting from free_all may still have incoming links into Free.

The following event removes any such links by deallocating the free list:

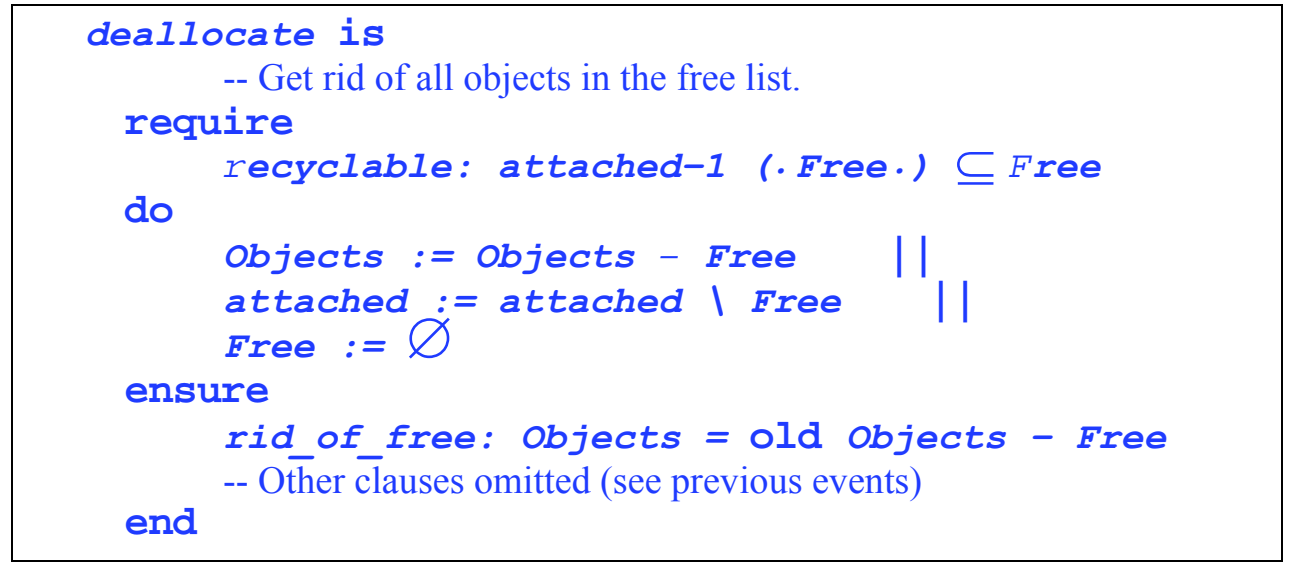

The precondition requires that incoming links into Free come only from Free itself, rather than from non-free Garbage elements (see [T49]). This is not the case for example on the figure of page 94 unless the two links going to $\mathrm{O} 1$ and $\mathrm{O} 2$ are taken away. Without this, objects = objects - Free would create Zombie links from objects to Unused; even if such links can only originate from Garbage, they stilll violate the Basic Object Constraint.

If, on the other hand, no links exist to Free objects except from Free itself, then the precondition of deallocate is satisfied. This is the case in particular when all garbage has been made free: 
(Free $=$ Garbage $) \Rightarrow$ recyclable

-- Here recyclable is the precondition of deallocate

(Proof: follows directly from [T44].) This means that it is legitimate to use deallocate just after free_all, thanks to the latter event's last postcondition clause, Garbage = Free.

\section{Incremental garbage collection}

The events collect_all and free_all represent a full GC cycle that removes all garbage. In a modern language implementation there must also be room for an incremental GC, which removes some garbage objects but not necessarily all.

This suggests that we need another event collect_some (Rejects) whose argument Rejects denotes a set of garbage objects. As a special case, collect_some (Garbage) will describe the same operation as free_all. At the other extreme, we can use collect some $(\{0\})$ to describe the collection of a single object $o$, or , in a non-GC-language, $\bar{a}$ programmer-controlled operation to free this object safely.

Here is collect_some in a form as close as possible to free_all:

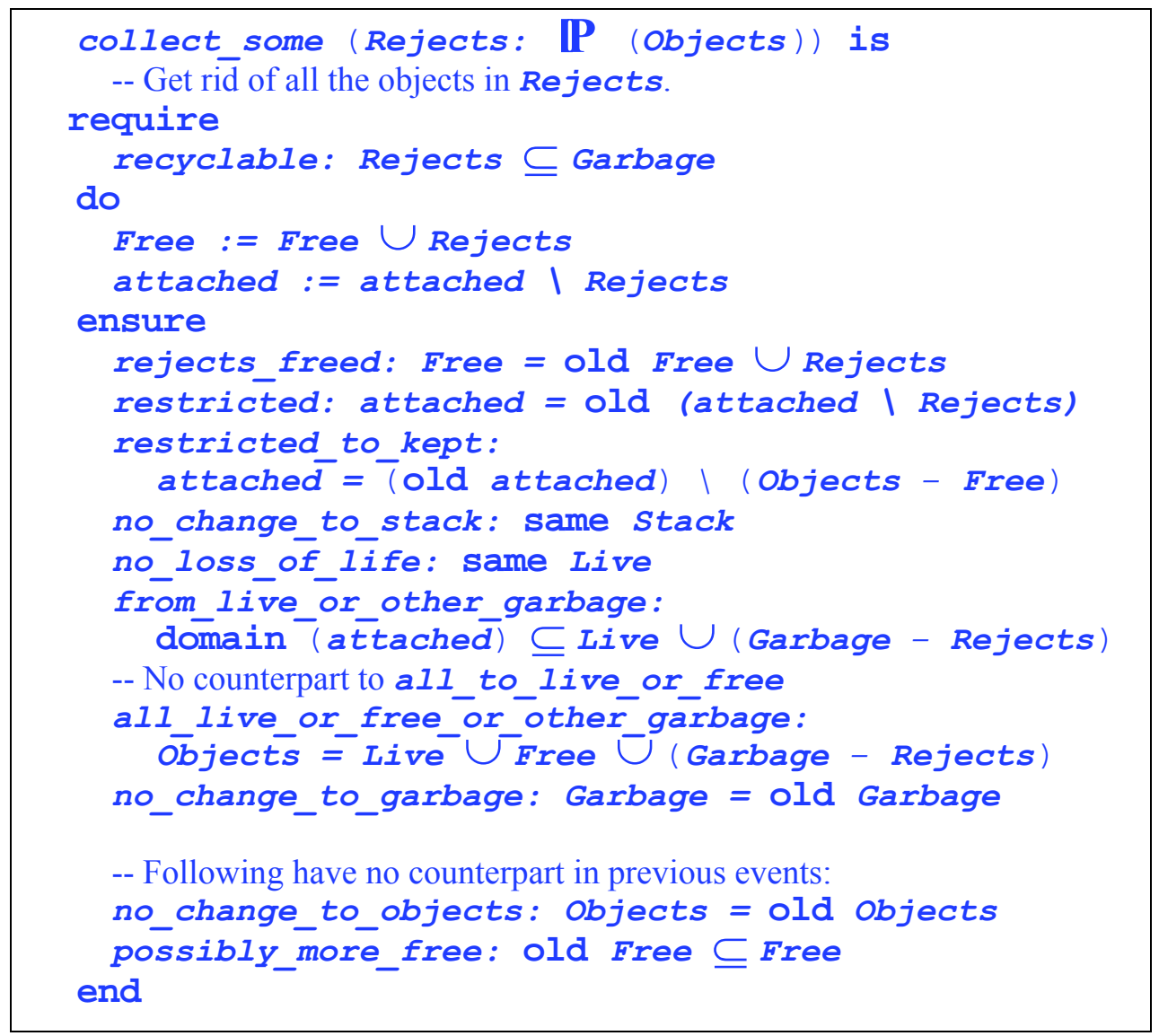


The replacement for attached is attached I Rejects, meaning: attached deprived of all pairs of the form $[i, j]$ where $i \in$ Rejects. The proofs are similar to those of the last two events and left to the reader; the key property is the precondition recyclable, without which we couldn't guarantee no_loss_of_life.

All clauses except the last two are counterparts of those of free_all, the same or weaker. The reason for using free_all as our model, rather than collect_all, is that collect some must be applicable to an arbitrary subset Rejects of Garbage: then it cannot remove the corresponding objects from objects, like collect_all, since as already noted any links into Rejects from the outside (which can only come from other Garbage) would yield Zombie links. So here the only possibility is a free_all-like behavior that moves the Rejects to the Free lists without removing them from the set of objects.

Removing the objects for good - sending them to Unused - means having a deallocate take place after collect_some, which is only possible if attached ${ }^{1}$ ( $\left.\operatorname{Rejects} \bullet\right) \subseteq$ Rejects to ensure the precondition of deallocate. (This is the case if Rejects is all of Garbage; indeed collect_some (Garbage) is the same as free_all.) Of course we could add this clause to the precondition of collect_some itself, enabling this event to remove the Rejects completely; but then the model ceases to be realistic since an incremental collection cycle would now need to find all the links into Rejects and hence to work on Garbage as a whole, whereas the very notion of incremental garbage collection implies that if you have spotted a few Rejects you can free them without having to traverse the rest of the Garbage. So the best collect_some can do in the general case is to move the Rejects to the Free list.

This reasoning is one of the principal justifications for introducing the notion of free list into the model.

\section{Recycling an object}

Even if prevented to return its Rejects to Unused, an incremental GC cycle will remove them from domain (attached). That's enough to make them available to the event allocate, which needs a suitable address for a new object. As we have seen ${ }^{15)}$, that doesn't mean an address outside of objects, just outside of both Live and (clause new_virginal) domain (attached). The postcondition clause from_live_or_other_garbage of collect_some ensures it.

As a consequence we may define an event that, by combining collect_some and allocate, frees an object and immediately reuses its address for a new object:

15 See discussion of new_virginal on page $\underline{90}$. 


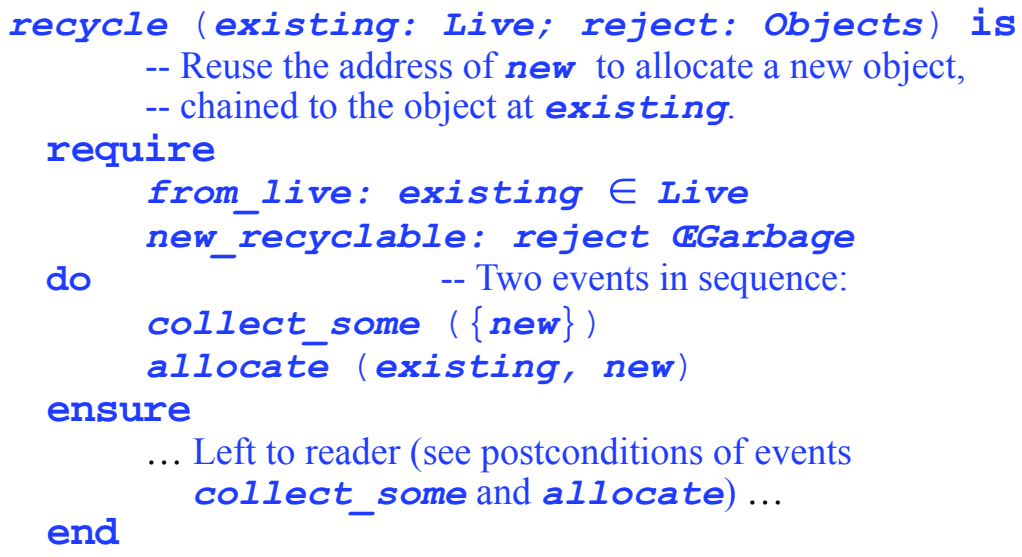

Its correctness requires that, as just noted, the precondition of allocate hold after collect some. The postcondition and the rest of the proof are left to the reader.

\section{Stack allocation}

The previous events had to do with objects allocated on the heap. In the execution of an objectoriented we also need a stack-based form of allocation, similar to pre-O-O techniques as present in Algol 60. The following event provides it; routine calls will use it for every local variable and by-value argument of a reference (non-expanded) type.

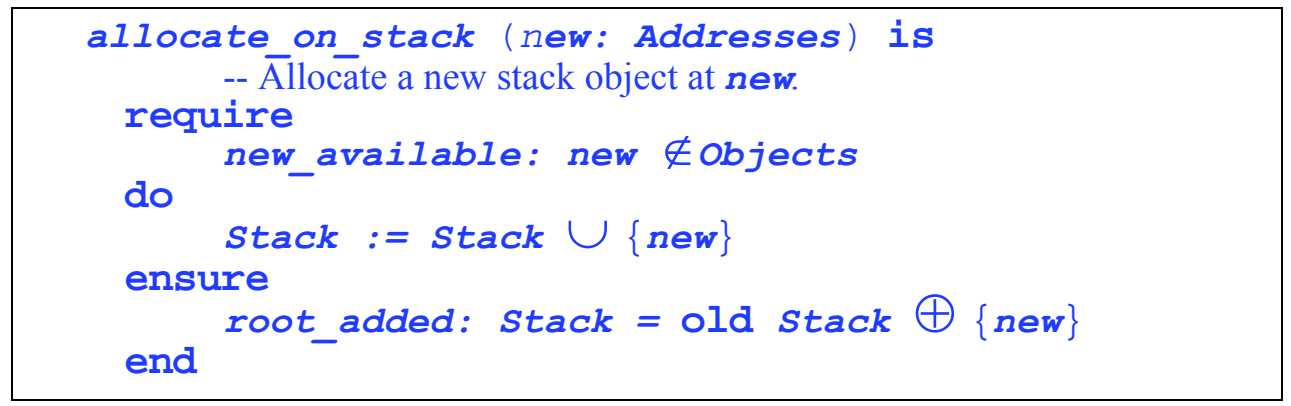

Proving the postcondition and the preservation of the invariants is easy. Note that here we cannot any more weaken the precondition new available to new $\notin$ Live and new $\notin$ domain (attached) as we did for the event allocate ${ }^{16)}$ : if new is a garbage object, other garbage objects may have links to it; then if we attempted to recycle it as a stack object we couldn't any more guarantee the postcondition, which requires new to be part of the stack and hence, from [T21], to admit no incoming link. So for the choice of new we exclude all currently allocated addresses, live as well as garbage. This matches the behavior of practical memory allocation schemes, which draw stack addresses and heap addresses from different address pools.!

One may similarly define an event free_from_stack (existing) that removes an element from the stack. This is left to the reader.

16 Clauses new_available and new_virginal, page $\underline{88}$; see also the discussion of the event collect_somē, starting on page $\underline{96}$. 


\section{REPRESENTING ADDRESSES}

The set of object addresses was specified as a set of integers (as introduced on page 77. .):

\section{[A1] Addresses: [F ( $\mathbb{N})$}

Do we indeed need to know what Addresses is? Not at this stage; we could proceed for a while without stating what Addresses is made of. One might even conjecture that this choice of a concrete set for Addresses betrays that the author of this discussion is a programmer, faithful to the usual mores of his species: implement first and maybe think later.

The choice is indeed the mathematical counterpart of what in software would be an implementation decision. But it seems justified if the goal is to build a useful model of the execution of programs on computers. Our computers have memories, and these memories have sequentially numbered cells. Hence the idea of defining Addresses as a set of integers. Even if early on we don't care about this aspect, it becomes relevant if our eventual aim is to formalize $\mathrm{O}-\mathrm{O}$ programs, or even just their GCs.

How much low-level an implementation decision this is depends on the intuitive semantics we attach to $\mathbb{N}$. Fortunately we can continue developing the model without choosing our exact level of abstraction:

- Under a "high-level" interpretation we may think of an abstract memory, where each cell (denoted by an integer from the domain of attached) contains an object - an instance of a class. For example the GC of versions 1 and 2 of ISE Eiffel relied on having all live objects linked together (through a hidden field added to every object); it also chained together in a "free list" all the dead objects it reclaimed. With such an interpretation every integer denotes not a physical address in the computer's memory but a position in a list of objects.

- We may also use a "low-level" interpretation and consider the integers to be the actual starting addresses of the objects' representation. One of the pleasant consequences of using relations - or, starting with the next article, possibly partial functions - is that they don't have to be total or surjective. So if an address doesn't correspond to the beginning of an actual object (in particular, if it is not a multiple of 4, assuming objects start on 32-bit word boundaries and our addresses are counted in bytes) it will simply not be in objects and hence will not denote any object.

This interpretation also yields (when combined with the Basic Object Constraint) an important property of the model: no references to subobjects. In Eiffel, even though the framework supports subobjects (through the "expanded" mechanism), a reference will always be attached to a first-level object, never to a subobject. The experience of early language and compiler versions showed that permitting references to subobjects complicates the GC and precludes some optimizations, for no significant expressive benefit. 
With the low-level interpretation we will most likely go further in our specification of Addresses and define it as the interval 1..memory high for some non-negative integer memory_high. From there one can start discussing in detail the properties of a memory management scheme. For example a GC of the mark-and-sweep kind needs in its "sweep" phase to traverse the whole memory - the interval 1.. memory_high.

This interpretation may also allow us to take into account the actual content of objects (their "expanded" fields), not just — as in the present discussion — the references they contain to other objects. The content of an object identified by the integer $n$ (assuming $n$ belongs to objects) is simply what's stored at a set of physical addresses starting at $n$ and bounded by next (n) - 1 where next $(n)$ is the next member of objects, if any.

At this stage, however, nothing forces us to disallow subobject references, or to choose the "high-level" interpretation or the "low-level" one, or any other. Their role is simply to reassure ourselves that the mathematical model is realistic.

\section{What has been postulated}

Here for convenience is a recapitulation of the assumptions made so far (marked originally with - signs): seven axioms, each postulating an element of a known set, and seven invariants postulating properties of these elements. They make up the basis of what we need to reason about run-time object structures. Every one of the events to be studied in later refinements. will have to preserve the invariants.

\begin{tabular}{llll|}
\hline$[\mathrm{A} 1]$ & Addresses: $\mathbb{F}(\mathbb{N})$ & Axioms \\
{$[\mathrm{A} 2]$} & Objects: $\mathbb{F}(\mathbb{N})$ & \\
{$[\mathrm{A} 5]$} & attached: $\mathbb{N} \leftrightarrow \mathbb{N}$ & \\
{$[\mathrm{A} 19]$} & Stack: $\mathbb{P}($ Addresses $)$ & \\
{$[\mathrm{A} 46]$} & Free: $\mathbb{F}(\mathbb{N})$ &
\end{tabular}

\begin{tabular}{lll|}
\hline$[\mathrm{I}]$ & $\begin{array}{l}\text { Objects } \subseteq \text { Addresses } \\
\text { attached }(\bullet \text { objects } \bullet) \subseteq \text { objects }\end{array}$ & Invariants \\
{$[\mathrm{I} 6]$} & Stack $\subseteq$ Objects - range (attached) & \\
{$[\mathrm{I} 20]$} & $\begin{array}{l}\text { Free } \subseteq \text { Garbage } \\
\text { Free } \cap \text { domain }(\text { attached })=\varnothing\end{array}$ & \\
\hline
\end{tabular}

